\title{
Relação entre escore cardíaco e o condicionamento físico de eqüinos da raça Mangalarga
}

\section{Relationship between heart score and physical conditioning in Mangalarga horses}

\author{
André Furugen Cesar de Andrade, ${ }^{*}$ Lílian Emy dos Santos Michima, ${ }^{* *}$ Letícia Andreza Yonezawa, ${ }^{* *}$ \\ Wilson Roberto Fernandes**
}

\begin{abstract}
Resumo
O tamanho do coração é, por definição, determinante da capacidade máxima do rendimento cardíaco e aeróbico do animal, e havendo a possibilidade de obter-se esta medida através do traçado eletrocardiográfico, verificou-se a relação entre escore cardíaco e a condição física de cavalos da raça Mangalarga. Foram examinados 70 animais com idade variando entre 11 meses a dez anos, distribuídos nos seguintes grupos: G1 (controle), com 30 animais sedentários, sendo 10 machos e 20 fêmeas; G2 (condicionados), constituído por 30 animais, 15 machos e 15 fêmeas, submetidos a um sistema regular de condicionamento por no mínimo 12 meses, e um grupo de dez animais (G3) constituído de potras que estavam iniciando o trabalho de condicionamento físico. Após a obtenção dos registros das derivações bipolares, calculou-se o escore cardíaco pela média aritmética da duração dos complexos QRS das derivações bipolares (I, II, III), considerando-se cinco complexos de cada uma, com o resultado dado em milissegundos (ms). A média do escore cardíaco encontrado no grupo G1 (97,10 $\pm 12,37$ $\mathrm{ms})$ foi significativamente menor que a média do grupo G2 $(129,07 \pm 9,28 \mathrm{~ms})$ com $p<0,05$. A média do grupo G3 $(89,0 \pm 11,7$ $\mathrm{ms})$ foi estatisticamente semelhante $(p>0,05)$ a do $G 1$ e menor $(p<0,05)$ que a encontrada no G2. No grupo $G 2$, não foi observada diferença significativa ( $p>0,05)$ entre os sexos, sendo a média das fêmeas condicionadas de 127,27 $\pm 7,98 \mathrm{~ms}$, enquanto a dos machos condicionados foi de 130,9 $\pm 10,4 \mathrm{~ms}$. No grupo dos animais não condicionados (G1), as fêmeas tiveram em média 97,4 $\pm 12,8 \mathrm{~ms}$ e os machos 96,6 $\pm 12 \mathrm{~ms}$. Encontrou-se uma correlação de média intensidade entre o EC e o perímetro torácico de 0,511 e entre o EC e a altura da cernelha de 0,490 . Os valores do perímetro torácico encontrados foram de $179,0 \pm 6,6$ no G1, de $187 \pm 6,1$ no G2, e de $164,6 \pm 9,9 \mathrm{~cm}$ para os pertencentes ao G3, enquanto para a altura da cernelha as medidas obtidas foram de 156,3 $\pm 4,8 \mathrm{em} \mathrm{G1}, 159,7 \pm 3,4 \mathrm{em} \mathrm{G2}$, e de 149,1 $\pm 5,5 \mathrm{~cm}$ em G3, com todos os valores diferindo significativamente quando comparados os grupos. Deste modo, a medida do escore cardíaco realmente apresentou uma relação com o estado de condicionamento dos cavalos estudados, assumindo valores muito maiores nos animais que realizavam treinamento, e pode-se provar que é possível supor a massa cardíaca por medidas indiretas como o perímetro torácico. Porém, deve-se ressaltar que estas são apenas ferramentas para predizer a condição física de um animal atleta, devendo o profissional atentar-se a todas as outras diversas variáveis que possam influenciar na saúde deste, tais como a função respiratória, a músculo-esquelética, a nutricional entre outras.
\end{abstract}

Palavras-chave: eqüinos, escore cardíaco, condicionamento físico, Mangalarga.

\begin{abstract}
Heart size, by definition, determines the animal's maximal cardiac and aerobic performance, and due to the possibility in obtaining this measure through electrocardiography, the relationship between heart score and physical conditioning in Mangalarga horses was verified. Seventy horses ranging from 11 month to 10 years old were examined, divided into three groups: G1 (control), with 30 adult sedentary horses (10 males and 20 females); G2 (conditioned), with 30 adult horses (15 males and 15 females) submitted to a regular training schedule for at least 12 months, and G3, with 10 fillies
\end{abstract}

beginning training. After the derivations registers were obtained, the heart score was calculated by the average of the QRS complexes duration in the bipolar derivations (I, II and III), considering five complexes in each one, with the result in milliseconds (ms). The mean heart score in G1 (97.10 \pm 12.37 $\mathrm{ms})$ was significantly smaller than G2 $(129.07 \pm 9.28 \mathrm{~ms})$, $\mathrm{P}<0.05$. G3 mean $(89.0 \pm 11.7 \mathrm{~ms})$ was statistically similar $(P>0.05)$ to $G 1$ and smaller $(P<0.05)$ than $G 2$. It was not observed significant difference $(P>0.05)$ between sexes in $G 2$, with a mean female heart score $127.27 \pm 7.98 \mathrm{~ms}$, whereas the male heart score was $130.9 \pm 10.4 \mathrm{~ms}$. In group G1 (sedentary animals) the females had a mean heart score of

\footnotetext{
* Departamento de Reprodução Animal - Faculdade de Medicina Veterinária e Zootecnia da Universidade de São Paulo

** Departamento de Clínica Médica - Faculdade de Medicina Veterinária e Zootecnia da Universidade de São Paulo

***Departamento de Clínica Médica - Faculdade de Medicina Veterinária e Zootecnia da Universidade Estadual Paulista Autor para correspondência: André Furugen Cesar de Andrade. E-mail: andrefc@usp.br Av. Duque de Caxias Norte, 225, CEP 13635-900, Caixa postal 23, Campus da USP, Pirassununga, SP, Brasil. Telefone: (019) 3565 - 4262 Fax:(019) $3565-4060$
} 
$97.4 \pm 12.8 \mathrm{~ms}$, and the males had $96.6 \pm 12 \mathrm{~ms}$. There was a linear correlation of median intensity between HS and thoracic perimeter $(r=0.511)$ and between HS and withers height $(r=0.490)$. Mean thoracic perimeter was $179.0 \pm 6.6$ for $\mathrm{G} 1,187 \pm 6.1$ for $\mathrm{G} 2$, and $164.6 \pm 9.9 \mathrm{~cm}$ for $\mathrm{G} 3$, whereas withers height means were $156.3 \pm 4.8$ for G1, $159.7 \pm 3.4$ for G2, and $149.1 \pm 5.5 \mathrm{~cm}$ for G3, with the values differing significantly between groups. Heart score presented a good relation with the conditioning status of the horses in this study, assuming higher values in training horses, and it was proved that it is possible to suppose the cardiac mass through indirect measurements as thoracic perimeter. However it is important to emphasize that these are tools to predict the physical conditioning from an athlete animal, and the professional must evaluate the other parameters that may influence the animal's health, as the respiratory, musculoskeletal and nutritional function as well.

Keywords: equine, heart score, physical conditioning, Mangalarga.

\section{Introdução}

O tamanho do coração é determinante da capacidade máxima do rendimento cardíaco e aeróbico do animal (Hodgson; Rose, 1994; Detweiler et al., 1996). Steel em 1963 (Steel, 1963 apud Moodie e Sheard, 1980) publicou o conceito de que o tamanho do coração poderia ser refletido pelo tempo de despolarização ventricular, representado pelo complexo QRS, este obtido no exame eletrocardiográfico.

Steel (1963 apud Moodie e Sheard, 1980), mostrou uma alta correlação $\left(r^{2}=0,89\right)$ entre a média de duração do complexo QRS nas derivações bipolares I, II, III do eletrocardiograma e o peso do coração de cavalos de corrida e de sela. Esta média aritmética dos valores do tempo de duração do complexo QRS nas três derivações bipolares foi denominada, por este autor, de escore cardíaco (EC).

Desde então, outros autores vêm desenvolvendo trabalhos com vistas a correlacionar o EC com o melhor rendimento em atividades físicas desenvolvidas por humanos, cães e cavalos (Physick-Sheard, 1999). Lannek e Rutquist (1951) chamaram atenção para tal fato, sendo que os maiores valores de duração eram observados nos eqüinos de esporte e principalmente no Puro-Sangue-Inglês (PSI).

O EC vem sendo relacionado com a performance do animal, assim como descrevem Rose et al. (1979), que afirmam que os animais mais velozes possuíam este índice maior que o dos outros competidores. Fato também relatado por Steel e Stewart (1974), que encontraram maiores valores nos animais vencedores das principais competições eqüestres da Austrália.

Um melhor rendimento é visto em cavalos com EC acima de 115 milissegundos (ms) (Bizzet et al., 1993; Nielsen; VibePetersen, 1980), já o baixo rendimento de animais condicionados é associada a valores de duração dos complexos eletrocardiográficos semelhantes àqueles de animais em início de treinamento (Rose; Davis, 1978).

Os resultados supracitados contradizem os achados de Leadon et al. (1982), que obtiveram uma correlação de baixa intensidade entre o escore cardíaco e a performance dos animais por eles estudados.

O EC é influenciado pela idade do animal, podendo ocorrer até 9,6 ms de acréscimo por ano, neste índice, em potros até a maturidade (Blakely; Blakely, 1995). Autores como Ayala et al. (1995) avaliaram que o complexo QRS, bem como outros valores do eletrocardiograma exibiram um aumento significativo dos seis meses aos dois anos de idade nos cavalos por eles estudados.

Há autores que indicam que o EC entre machos e fêmeas não difere (Steel; Stewart, 1974; Ayala et al., 1995), enquanto outros encontraram diferenças nos valores de tempo de duração do complexo QRS, e embasaram estes achados na existência de diferenças fisiológicas entre machos e fêmeas das diferentes espécies (Steel et al., 1976; Nielsen; VibePetersen, 1980).

Pesquisadores como Kubo, Senta, e Sugimoto (1974) avaliando a relação entre o período de treinamento e a hipertrofia cardíaca em animais mortos, observaram que os animais submetidos a treinamento possuíam uma maior massa cardíaca quando comparados aos não treinados. Neste mesmo trabalho notou-se que os animais atletas submetidos a alguns meses de descanso tiveram, em média, uma massa cardíaca inferior à dos cavalos bem condicionados. Este resultado levou estes pesquisadores a presumirem que o exercício leva o cavalo a uma hipertrofia cardíaca, enquanto o descanso leva a uma atrofia, mostrando que o condicionamento físico do animal pode ser avaliado pelo tamanho do coração.

Logo, a determinação do escore cardíaco em cavalos que participam de provas esportivas é de grande valor, a fim de evitar que animais em más condições físicas (escore cardíaco menor que $100 \mathrm{~ms}$ ) sofram alguma lesão devido à fadiga causada pelo esforço (Illera; Illera, 1987).

Partindo destes relatos e sendo o Mangalarga uma raça nacional com grande participação em esportes eqüestres, foi realizado o presente trabalho, para determinar o estado do condicionamento físico dos animais através da metodologia do escore cardíaco.

\section{Objetivos}

Este trabalho teve por objetivos verificar a relação entre o escore cardíaco e a condição física de cavalos da raça Mangalarga, comparando o traçado eletrocardiográfico de animais submetidos a uma rotina de treinamento com animais não submetidos a esforço físico, determinar valores de escore cardíaco para animais desta raça com condicionamento físico para participar de eventos esportivos e caracterizar correlações entre o escore cardíaco e medições de fácil obtenção como perímetro torácico e altura de cernelha.

\section{Material e método}

Foram examinados 70 eqüinos da raça Mangalarga, distribuídos entre machos e fêmeas clinicamente hígidos, de 11 meses a dez anos de idade, pesando de 250 a $600 \mathrm{~kg}$, com manejos nutricional, sanitário e higiênico similares.

Os animais foram divididos em três grupos: G1 (controle), composto por 10 machos e 20 fêmeas que não desenvolviam atividade física com idade entre 4 e 10 anos; e peso variando de 450 a $600 \mathrm{~kg}$; G2 (condicionados), com 15 machos e 15 fêmeas que eram submetidos a um sistema regular de condicionamento por no mínimo 12 meses, com idade 
variando de 4 a 10 anos, com peso variando de 450 a $600 \mathrm{~kg}$; e G3, constituído de dez fêmeas que estavam sendo iniciadas ao condicionamento físico, com idade variando de 11 a 20 meses, e peso variando de 250 a $350 \mathrm{~kg}$.

O traçado eletrocardiográfico foi obtido com o uso de um aparelho de eletrocardiograma ECG-6 (ECAFIX), seguindo a metodologia de Fregin (1982), registrado e padronizado com sensibilidade de $1 \mathrm{~cm}=1 \mathrm{mV}$ e velocidade de $25 \mathrm{~mm} / \mathrm{s}$. Foram realizadas as derivações bipolares (I, II, III) e as unipolares aumentadas (aVR, aVL, aVF), a fim de diagnosticar possíveis processos mórbidos que poderiam acometer os animais. Os registros eletrocardiográficos foram avaliados quanto à normalidade para a raça, levando-se em conta os padrões citados por Vincenzi (1995).

Após a obtenção dos registros das derivações bipolares, calculou-se o escore cardíaco, considerou-se a duração de cinco complexos QRS por derivação bipolar (I, II, III) (Figuras 1,2 e 3). O resultado da soma de todos os complexos QRS foi dividido por 15 , sendo o produto desta equação o escore cardíaco, em milissegundos (ms), do animal examinado.
Foi realizada a estatística descritiva de todas as variáveis estudadas e a análise de correlação de Pearson entre as diversas variáveis, considerando-se o nível de probabilidade de $5 \%$.

Dos valores obtidos desenvolveu-se um estudo estatístico através do teste-t para amostras independentes, adotando um nível de significância de $5 \%$ para a comparação das variáveis estudadas entre os diversos grupos.

\section{Resultados}

O valor médio do escore cardíaco encontrado nos animais pertencentes ao grupo $\mathrm{G} 1 \quad(97,10 \pm 12,37 \mathrm{~ms})$ foi estatisticamente menor $(p<0,05)$ que a média nos animais do grupo G2 (129,07 \pm 9,28 ms). Já a média do grupo G3 $(89,0 \pm 11,7 \mathrm{~ms})$ foi semelhante à do $\mathrm{G} 1$ e menor que a encontrada no G2, para $p<0,05$ (Tabela 1, Gráfico1).

Não houve diferença significativa $(p>0,05)$ entre machos e fêmeas de ambos os grupos (G1 e G2) (Tabela 2). A média das fêmeas condicionadas foi de 127,27 $\pm 7,98$ ms enquanto a dos machos condicionados foi de 130,9 $\pm 10,4$ ms. Já no

\section{Derivações bipolares mensuradas para a obtenção do escore cardíaco}

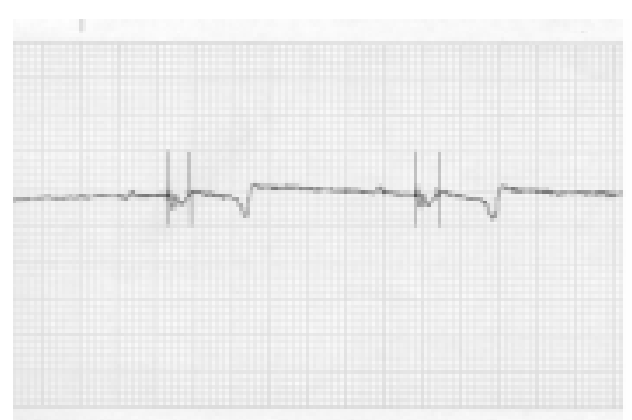

Figura 1: Duração do complexo QRS em DI (120 ms)

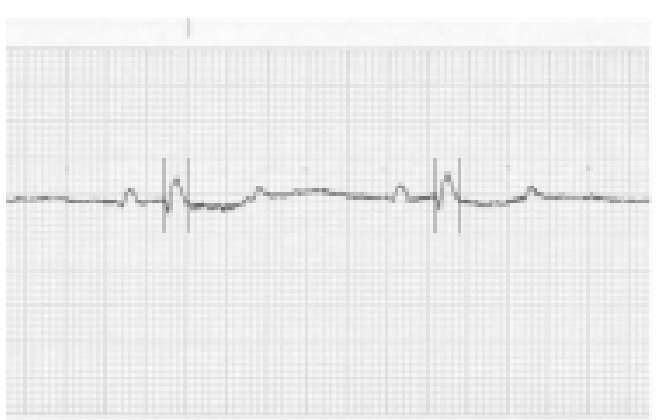

Figura 2: Duração do complexo QRS em DII (120 ms)

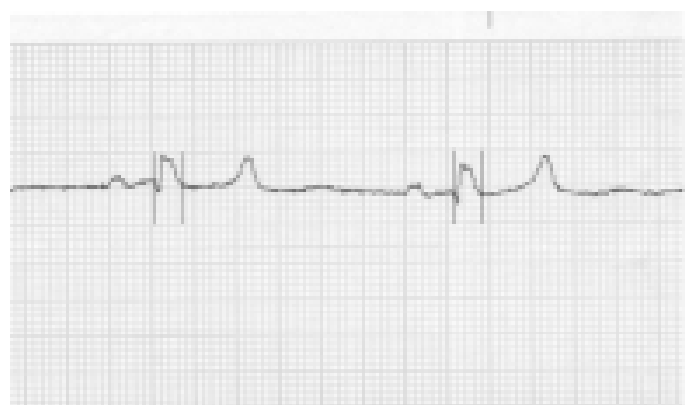

Figura 3: Duração do complexo QRS em DIII (120 ms)

Os valores referentes à altura de cernelha e perímetro torácico foram realizados com o uso de uma fita métrica, graduada em centímetros, com valores variando de 0 a 201. grupo dos animais não condicionados, as fêmeas tiveram em média $97,4 \pm 12,8 \mathrm{~ms}$ e os machos $96,6 \pm 12 \mathrm{~ms}$, como pode ser observado no Gráfico 2 e na Tabela 2. 
Tabela 1: Escore cardíaco, idade, peso, perímetro torácico e altura de cernelha de eqüinos da raça Mangalarga, com diferentes graus de condicionamento físico. São Paulo, 2004

\begin{tabular}{l|ccc}
\hline & G1 & G2 & G3 \\
\cline { 2 - 4 } Escore cardíaco $(\mathrm{ms})$ & $97,1 \pm 12,37^{\mathrm{A}}$ & $129,07 \pm 9,28^{\mathrm{B}}$ & $89,0 \pm 11,7^{\mathrm{A}}$ \\
Idade (anos) & $4-10^{\mathrm{C}}$ & $4-10^{\mathrm{C}}$ & $11 \mathrm{~m}-20 \mathrm{~m}^{\mathrm{D}}$ \\
Peso $(\mathrm{kg})$ & $450-600^{\mathrm{E}}$ & $450-600^{\mathrm{E}}$ & $250-350^{\mathrm{F}}$ \\
Perímetro torácico $(\mathrm{cm})$ & $179,0 \pm 6,6^{\mathrm{G}}$ & $187,0 \pm 6,1^{\mathrm{H}}$ & $164,6 \pm 9,9^{\mathrm{L}}$ \\
Altura de cernelha $(\mathrm{cm})$ & $156,3 \pm 4,8^{\mathrm{J}}$ & $159,7 \pm 3,4^{\mathrm{L}}$ & $149,1 \pm 5,5^{\mathrm{M}}$ \\
$\mathrm{n}$ & 30 & 30 & 10 \\
\hline
\end{tabular}

Caracteres diferentes na mesma linha indicam diferença estatística significante $(p<0,05)$ entre os grupos

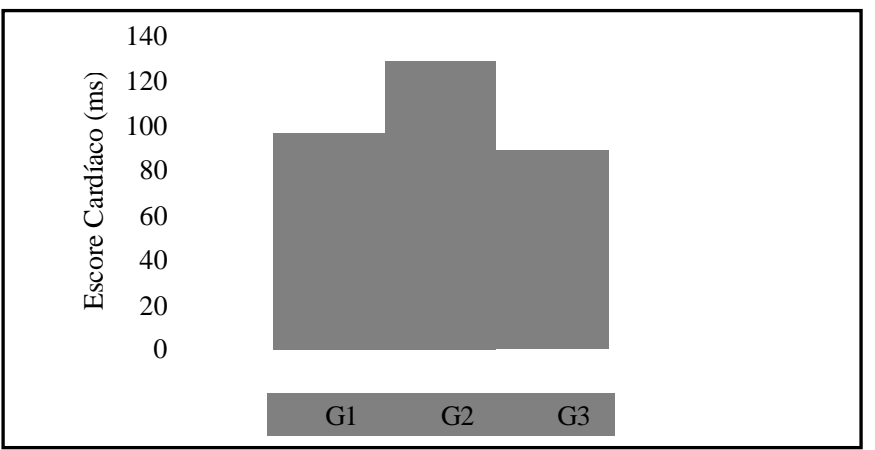

Gráfico 1: Valores de escore cardíaco nos diferentes grupos (G1, G2, G3)

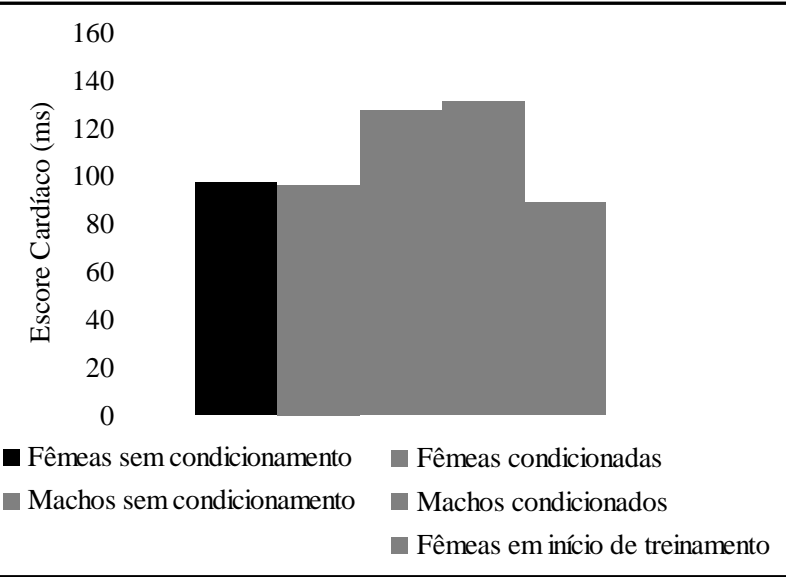

Gráfico 2: Valores obtidos nas derivações bipolares diferindo-se sexo e idade

Tabela 2: Escore cardíaco (em milissegundos) de eqüinos da raça Mangalarga, de acordo com o sexo e com diferentes graus de condicionamento físico. São Paulo, 2004

\begin{tabular}{l|cc}
\hline \multirow{2}{*}{ Fêmeas } & G1 & G2 \\
\cline { 2 - 3 } Machos & $97,4 \pm 12,8^{\mathrm{A}}$ & $127,27 \pm 7,98^{\mathrm{B}}$ \\
& $96,6 \pm 12,0^{\mathrm{A}}$ & $130,9 \pm 10,4^{\mathrm{B}}$ \\
\hline
\end{tabular}

Caracteres diferentes indicam diferença estatística significante $(p<0,05)$ entre os grupos
Com os dados obtidos ainda foi possível observar uma correlação positiva de média intensidade $(r=0,511)$ entre 0 perímetro torácico e o escore cardíaco, bem como entre altura de cernelha e o escore cardíaco $(r=0,490)$.

Os valores de perímetro torácico e altura de cernelha quando comparados estatisticamente entre os grupos, apresentavam valores que diferiram significativamente $(p<0,05)$ de grupo para grupo, sendo que os maiores valores foram encontrados no grupo dos animais condicionados. Os valores do perímetro torácico encontrados foram de $179,0 \pm 6,6$ no G1, de 187,0 \pm 6,1 no G2, e de 164,6 \pm 9,9 cm para os pertencentes ao G3, enquanto para a altura de cernelha as medidas obtidas foram de $156,3 \pm 4,8$ em G1, 159,7 $\pm 3,4$ em G2, e de $149,1 \pm 5,5 \mathrm{~cm}$ em G3.

\section{Discussão}

Os resultados encontrados mostraram que os cavalos pertencentes ao grupo de animais não condicionados (G1$97,10 \pm 12,37)$, ou seja, animais que não participam de competições, possuíam o valor médio do EC abaixo de 100 ms, o que vem de encontro aos achados de Illera e Illera (1987). Estes autores citam que os animais com condicionamento físico para participar de competições com necessidade de esforço físico, devem ter valores de EC maiores que $100 \mathrm{~ms}$.

Os EC encontrados em G1(97,10 $\pm 12,37)$ indicam que os animais estão com o condicionamento físico inadequado, fato esperado, pois este grupo era constituído de animais mantidos a pasto e que não estavam participando de nenhum programa de treinamento físico. O que corrobora com os achados de Kubo, Senta, e Sugimoto (1974), já que os cavalos do G1 já haviam passado por um período de treinamento. Estes animais ao serem examinados estavam sem efetuar trabalho de condicionamento há mais de um ano. Logo, como era esperado, estes cavalos tiveram valores de EC menores do que o grupo de animais condicionados, o que indica um processo de atrofia da musculatura do coração destes animais.

Os valores de EC encontrados em animais no início de treinamento (G3), como era esperado, foram iguais $(p<0,05)$ ao dos animais do G1, fato não inusitado; já os animais do G3 são potras que devem ainda acrescer e muito os valores do escore cardíaco como citado por Blakely e Blakely (1995). Os resultados do presente experimento mostram que o exercício leva a uma hipertrofia da musculatura cardíaca (aumento do coração), mas o fator idade é importante e leva a discrepâncias no valor do EC. É importante frisar relatos citando que, com a idade este índice apresenta um acréscimo de até $30 \mathrm{~ms}$ do nascimento até os três anos de idade (Steel, 1974), fato confirmado nas citações de Ayala et al. (1995), os quais observaram que os valores mensuráveis pelo eletrocardiograma tendem a aumentar com a idade.

Os valores de escore cardíaco nos animais condicionados (G2) foram semelhantes aos comunicados por Rose et al. (1979), Steel e Stewart (1974), Nielsen e Vibe-Petersen (1980), Rose et al. (1980), Bizzet et al. (1993), entre outros autores que, analisando em seus trabalhos apenas o eletrocardiograma de animais que praticavam esporte (condicionados), encontraram valores deste índice acima de 100 ms em todos os cavalos analisados.

Os resultados adquiridos indicam que há um maior desenvolvimento da massa cardíaca em animais atletas (G2), 
levando em consideração a correlação de alta intensidade entre o escore cardíaco e o peso do coração descrito por Steel (1963 apud Moodie e Sheard, 1980). Estes resultados coincidem com o descrito por alguns autores que, trabalhando com outras raças de eqüinos, verificaram que o escore cardíaco e outras medidas do traçado eletrocardiográfico apresentam um aumento significativo de seus valores após um período de treino, indicando que o treinamento leva a um aumento da musculatura cardíaca (Wyatt; Mitchell, 1974; Lonsdale et al., 1998; Constable et al., 2000).

Os valores do EC de alguns animais avaliados no presente experimento apresentaram-se acima dos citados na literatura em cavalos de corrida, como os encontrados por Bizzet et al. (1993), os quais encontraram o valor de escore cardíaco de 120 ms no cavalo Puro-Sangue-Inglês (PSI) de melhor performance atlética do grupo estudado. Porém estes achados não mostram que os animais da raça Mangalarga são mais velozes ou mais condicionados que um PSI de corrida. A explicação dos dados obtidos deve estar na conformação física da raça, que inclui o tamanho, o comprimento e a largura torácica que podem alterar a disposição do eixo cardíaco, assim alterando o traçado eletrocardiográfico, o que deve ser analisado em trabalhos posteriores.

O resultado de correlação positiva entre perímetro torácico e EC $(0,511)$ é importante, já que é uma medição de fácil obtenção e realizada com um material de baixo custo e fácil aquisição. A discussão que se segue à análise deste dado parte do princípio que na Medicina Veterinária é da prática obter-se o peso aproximado do animal pela mensuração do perímetro torácico.

Neste trabalho os resultados mostraram-se, por vezes, contraditórios aos encontrados na literatura, nos quais os autores trabalharam correlacionando o peso vivo de animais e humanos com o escore cardíaco. Autores como Steel e Stewart (1974) discordam com os resultados obtidos neste experimento, já que citam que não há correlação entre a massa do corpo de atletas humanos e o escore cardíaco, opinião coincidente com a de Hanson et al. (1994), que obtiveram seus valores trabalhando com cavalos de tração, animais que, em média, pesam $300 \mathrm{~kg}$ a mais do que os animais examinados neste trabalho.

Porém Steel et al. (1976) contradizem o resultado anterior publicado por Steel e Stewart (1974) ao publicarem que existe uma correlação de alta intensidade $(0,71)$ entre a massa corporal e o peso do coração em cachorros da raça Galgo, bem como em humanos e cavalos, resultados que em parte coincidem com o encontrado neste experimento, já que foi relatada uma correlação de média intensidade entre o perímetro torácico e o escore cardíaco.

Quando comparada à relação do sexo do animal com o índice escore cardíaco, o presente trabalho não encontrou diferenças significativas, o que coincide com os valores descritos por Steel e Stewart (1974) e Ayala et al. (1995), e colocam em dúvida os achados de certos autores que encontraram diferenças significativas nos traçados eletrocardiográficos de machos e fêmeas da espécie canina e eqüina, que atribuíam esta diferença a padrões fisiológicos que seriam diferentes entre os sexos (Steel et al., 1976; Nielsen; Vibe-Petersen, 1980).

\section{Conclusão}

A medida do escore cardíaco tem relação com o estado de condicionamento dos cavalos estudados, assumindo valores muito maiores nos animais que realizavam treinamento.

Pode-se atribuir um maior escore cardíaco a cavalos da raça Mangalarga que possuem um maior perímetro torácico.

Porém, deve-se ressaltar que o índice escore cardíaco é apenas uma ferramenta para predizer a condição de um animal, devendo o profissional atentar-se a todas as outras variáveis que possam influenciar na saúde deste, tais como a função respiratória, a músculo-esquelética, nutricional entre outras.

\section{Agradecimentos}

À Fundação de Amparo à Pesquisa do Estado de São Paulo - FAPESP, pela bolsa de iniciação científica concedida.

\section{Referências}

AYALA, I.; MONTES, A.;BERNAL, L. J.; SANDOVAL, J. A., GUTIERREZ, C. Eletrocardiographic values in Spanish-bred horses of different ages. Australian Veterinary Journal, v. 72, n. 6, p. 225-226, 1995.

BLAKELY, J. A.; BLAKELY, A. A. The accuracy of predicting thoroughbred heart scores. New Zealand Veterinary Journal, v. 43, p. 57-59, 1995.

BIZZET, M.; CORAZZA, M.; PECCHIA, B.; CURRARINI, L. L'eletrocardiogramma nel cavallo atleta a riposo, com particolare riferimento al punteggio cardíaco. Annali della Facoltá di Medicina Veterinaria di Pisa, v. 46, p. 121-132, 1993.

CONSTABLE, P. D.; HINCHCLIFF, K. W.; OLSON, J. L.; STEPIEN, R. L. Effects of endurance training on standard and signal-avareged electrocardiograms of sled dogs. American Journal Veterinary Research, v. 61, p. 582-588, 2000.

DETWEILER, D.K.; RIEDESEL, D. H.; KNIGHT, D. H. Atividade Mecânica do Coração. In: SWENSON, M. J.; REECE, W. O. (Ed.). DUKES: fisiologia dos animais domésticos. Rio de Janeiro: Guanabara Koogan, 1996. p. 133-156.

FREGIN, G. F. Electrocardiography. The Veterinary Clinics of North America. Equine Practice, v. 1, n. 2, p. 419-432, 1985.

HANSON, C.M.; KLINE, K. H.; FOREMAN J. H. Measurements of heart scores and heart weights in horses of two different morphic body types. Comparative Biochemistry and Physiology, v. 108A, p. 175178, 1994.

HODGSON, D. R.; ROSE, R. J. Evaluation of performance potential. In: . The athletic horse: principles and practice of equine sports medicine. Philadelphia: W. B. Saunders, 1994, p. 231-244.

ILLERA, J. C.; ILLERA, M. Electrocardiography and heart score of horses competing in an endurance ride. Australian Veterinary Journal, v. 64, n. 3, p. 88-89, 1987.

KUBO, K.; SENTA, T.; SUGIMOTO, O. Relationship between training and heart in the thoroughbred racehorse. Experimental Reporting of Equine Health Laboratory, n. 11, p. 87-89, 1974. 
LANNEK, N.; RUTQUIST, L. Normal area of variation for the electrocardiogram of horses. Nordisk Veterinaer Medicin, v. 3, p. 1094-1117, 1951.

LEADON, D. P.; CUNNINGHAM, E. P.; MAHON, G. A.; TODD, A. J. Heart score and performance ability in the United Kingdom. Equine Veterinary journal, v. 14, n. 1, p. 89-90, 1982.

LONSDALE, R. A.; LABUC, R. H.; ROBERTSON, I. D. Echocardiographic parameters in training compared with non-training greyhounds. Veterinary Radiology and Ultrasound, v. 39, n. 4, p. 325-330, 1998.

MOODIE, E. W.; SHEARD, R. P. The use of electrocardiography to estimate heart weight and predict performance in the racehorse. Australian Veterinary Journal, v. 58, n. 11, p. 557-558, 1980.

NIELSEN, K.; VIBE-PETERSEN, G. Relationship between QRS-duration (heart score) and racing performance in trotters. Equine Veterinary Journal, v. 12, n. 2, p. 81-84, 1980.

PHYSICK-SHEARD, P. W. Heart score theory. In: CALAHAN, P. T.; MAYHEW, I. G.; MERRITT, A. M.; MOORE, J. N. (Ed.). Equine medicine and surgery. St. Louis: Mosby, 1999, p. 336-337.

ROSE, R. J.; BACKHOUSE, J. W.; ILKIN, J. E. Electrocardiography and haematology of horses competing in a three-day event. Australian Veterinary Journal, v. 56, n. 7, p. 318-320, 1980.

ROSE, R. J.; DAVIS, P. E. The use of electrocardiography in the diagnosis of poor racing performance in the horse. Australian Veterinary Journal, v. 54, n. 2, p. 51-56, 1978.
ROSE, R. J.; ILKIW, J. E.; HODGSON, D. Electrocardiography, heart score and haematology of horses competing in an endurance ride. Australian Veterinary Journal, v. 55, n. 6, p. 247-250, 1979.

STEEL, J. D.;BEILHARZ, R. G.; STEWART, G. A.; GOODDARD, M. The inheritance of heart score in racehorses. Australian Veterinary Journal, v. 53, n. 7, p. 306-309, 1977.

STEEL, J. D.; STEWART, G. A. Electrocadiography of the horse and potential performance ability. Journal of South African Veterinary Association, v. 45, n. 4, p. 269-271, 1974.

STEEL, J. D.; TAYLOR, R. I.; DAVIS, P. E.; STEWART, G. A.; SALMONS, P. W. Relationships between heart score, heart weight and body weight in greyhound dogs. Australian Veterinary Journal, v. 52, p. 561-564, 1976.

STEEL, J. D. 1963 apud MOODIE, E. W.; SHEARD, R. P. 1980, p. 557. STEWART, G. A. The heart score theory in the racehorse. Australian Veterinary Journal, v. 57, n. 9, p. 422-428, 1981.

VINCENZI, R. C. Determinação dos parâmetros eletrocardiográficos de eqüinos da raça Mangalarga, criados no Estado de São Paulo. 1995. 71 f. Dissertação (Mestrado em Ciências) - Faculdade de Medicina Veterinária e Zootecnia, Universidade de São Paulo, São Paulo.

WYATT, H. L.; MITCHELL, J. H. Influences of physical training on the hearts of dogs. Circulation Research, v. 35, p. 883-889, 1974. 